\title{
Pre-exposure prophylaxis awareness and interest among participants in a medications for addiction treatment program in a unified jail and prison setting in Rhode Island
}

\author{
Meghan Peterson ${ }^{\mathrm{a}, \mathrm{b}, *}$, Alexandria Macmadu ${ }^{\mathrm{b}, \mathrm{c}}$, Ashley Q. Truong ${ }^{\mathrm{b}}$, Josiah Rich ${ }^{\mathrm{b}, \mathrm{c}, \mathrm{d}}$, \\ Kimberly Pognon $^{\text {b }}$, Mark Lurie ${ }^{c}$, Jennifer G. Clarke ${ }^{\text {e,f }}$, Lauren Brinkley-Rubinstein ${ }^{\text {g, }}$,

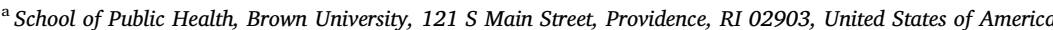 \\ ${ }^{\mathrm{b}}$ Center for Prisoner Health and Human Rights, 8 3rd Street, Providence, RI 02906, United States of America \\ ${ }^{\mathrm{c}}$ Department of Epidemiology, School of Public Health, Brown University, 121 S Main Street, Providence, RI 02903, United States of America \\ ${ }^{\mathrm{d}}$ Department of Emergency Medicine, Warren Alpert Medical School of Brown University, 222 Richmond St, Providence, RI 02903, United States of America \\ ${ }^{\mathrm{e}}$ Rhode Island Department of Corrections, 40 Howard Ave, Cranston, RI 02920, United States of America \\ ${ }^{\mathrm{f}}$ Departments of Medicine and Obstetrics and Gynecology, Alpert Medical School of Brown University, 222 Richmond St, Providence, RI 02903, United States of America \\ ${ }^{g}$ Department of Social Medicine, University of North Carolina, 333 S Columbia St, Chapel Hill, NC 27516, United States of America \\ ${ }^{\mathrm{h}}$ Center for Health Equity Research, University of North Carolina, 335 S Columbia St, Chapel Hill, NC 27516, United States of America
}

A R T I C L E I N F O

Keywords:

Pre-exposure prophylaxis (PrEP)

Incarceration

Opioid use disorder

Qualitative research

\begin{abstract}
A B S T R A C T
People who are incarcerated are at increased risk for HIV (human immunodeficiency virus) acquisition upon release, and one possible intervention for prevention is the use of pre-exposure prophylaxis (PrEP) upon release. The present study assessed HIV risk perceptions as well as PrEP awareness and interest among 39 people who were incarcerated and enrolled in a structured Medication for Addiction Treatment (MAT) program at the Rhode Island Department of Corrections using semi-structured, qualitative interviews. Analysis was conducted using a generalized, inductive method in NVivo 12. While PrEP awareness was low across the study sample, some participants were interested in PrEP uptake or learning more about PrEP after they were provided with an overview of it. PrEP interest strongly related to current perceived HIV risk. Potential barriers included side effects, adherence, and reluctance to take medications in general. MAT programs for people who are criminal justice (CJ) involved may serve as useful linkage spaces to PrEP information, access, and retention.
\end{abstract}

\section{Introduction}

People who are incarcerated are at increased risk for HIV (human immunodeficiency virus) acquisition (Wohl, 2016), and the prevalence of HIV in incarcerated populations is approximately five times higher than in the general population (Maruschak, 2012). Populations such as Black men and people who use drugs-who are at greater risk for HIV acquisition-have historically been incarcerated in the United States through "mass incarceration" at rates that are disproportionate to the general population (Wohl, 2016). Furthermore, in the period after release from incarceration, engagement in behaviors such as multiple, overlapping partnerships and sex without condoms can increase HIV risk (Morrow and The Project START Study Group 1, 2009). Social relationships that may have previously protected against HIV acquisition are often disrupted by stressors related to community reentry, putting individuals at risk upon release from incarceration (Khan et al., 2011).

One possible intervention for criminal justice (CJ) involved populations is the use of pre-exposure prophylaxis (PrEP) to reduce HIV risk after release from incarceration (Brinkley-Rubinstein, Peterson et al., 2018). PrEP is an effective, biomedical intervention consisting of tenofovir-emtricitabine taken orally daily among HIV negative individuals to prevent infection (Anderson et al., 2012; Baeten et al., 2012; Choopanya et al., 2013). Daily PrEP use reduces the risk of HIV acquisition from sexual transmission by over $90 \%$, and from injection drug use by over 70\% (Centers for Disease Control and Prevention and US Public Health Service, 2018). Adherence to PrEP during periods of high individual risk behaviors increases the efficacy of PrEP (Grant

\footnotetext{
* Corresponding author at: Brown University School of Public Health, 121 South Main Street, Providence, RI 02901, Box G-S121-3, United States of America.

E-mail addresses: Meghan_peterson@alumni.brown.edu (M. Peterson), alexandria_macmadu@alumni.brown.edu (A. Macmadu), ashley_truong@alumni.brown.edu (A.Q. Truong), jrich@lifespan.org (J. Rich), kimberly.pognon@lifespan.org (K. Pognon), mark_lurie@brown.edu (M. Lurie), jennifer.clarke@doc.ri.gov (J.G. Clarke), lauren_brinkley@med.unc.edu (L. Brinkley-Rubinstein).
} 
et al., 2014). However, PrEP uptake, particularly among groups at high risk in the United States, has been slow (Kirby \& Thornber-Dunwell, 2014), with only about $10 \%$ of the population who might benefit uptaking PrEP medication (Mayer, Chan, Patel, Flash, \& Krakower, 2018). It is estimated that approximately 78,000 Americans over the age of 16 held a PrEP prescription in 2016 (Ya-lin, Zhu, Smith, Harris, \& Hoover, 2018). Most people taking PrEP were white and male, suggesting that more equitable implementation to women and ethnic/racial minorities is needed (Ya-lin et al., 2018).

In addition to taking a daily pill, taking PrEP includes regular engagement in care. Centers for Disease Control (CDC) guidelines dictate that providers should test for HIV status prior to uptake of PrEP, and that HIV infection should be assessed every three months to ensure that individuals with incident infections do not remain on PrEP. People taking PrEP should receive HIV counseling, behavioral risk reduction support, side effect assessment, and STI testing every three months (Centers for Disease Control and Prevention and US Public Health Service, 2018). Upon uptake, maximum concentrations for effective prevention are reached in blood after approximately twenty days of oral dosing, reached in rectal tissue after seven days, and reached in cervicovaginal tissue after twenty days (Centers for Disease Control and Prevention and US Public Health Service, 2018). Side effects vary, but most commonly include nausea, headaches, rash, and flatulence (Stekler et al., 2016).

While few studies have explored the use of PrEP in correctional settings, there is evidence that CJ-involved populations are often unaware of PrEP but express interest once they learn more about it. Studies among men who have sex with men who were incarcerated in Rhode Island and women who were criminal justice-involved in San Francisco have detailed these findings (Brinkley-Rubinstein, Peterson, et al., 2018; Dauria et al., 2017). Qualitative research among men who have sex with men (MSM) who were incarcerated in Rhode Island detailed how, despite interest, perceived barriers may impede both PrEP uptake and adherence. These barriers include institutional distrust, medication costs, anticipated partner disapproval, transportation access, and compounding impacts of multiple hardships upon release (BrinkleyRubinstein, Peterson, et al., 2018; Peterson, Nowotny, Dauria, Arnold, \& Brinkley-Rubinstein, 2019).

Given that people who inject drugs (PWID) are at increased risk for HIV acquisition, including people with opioid use disorder (OUD) (Mathers et al., 2008), PrEP may be suitable for this subpopulation. Prior work has documented willingness to uptake PrEP among some people who inject drugs (Escudero et al., 2015; Kuo et al., 2016; Sherman et al., 2019; Stein, Thurmond, \& Bailey, 2014; Walters, Reilly, Neaigus, \& Braunstein, 2017). However, interest among people at risk for HIV infection may be limited due to aversion to biomedical interventions (Guise, Albers, \& Strathdee, 2017). Past work has also demonstrated that PrEP is acceptable to people who are engaged in substance use treatment, although barriers such as accessibility, need for daily adherence, and stigma exist (Shrestha, Altice, Huedo-Medina, Karki, \& Copenhaver, 2017; Shrestha \& Copenhaver, 2018).

Similarly, because of the high prevalence of people with OUD in the criminal justice system, recent interventions have examined the provision of medication for addiction treatment (MAT)—clinically effective regimens of medications such as methadone, suboxone, or naltrexone, combined with behavioral therapy-to people in CJ settings (Gordon et al., 2014; Rich et al., 2015; Zaller et al., 2013). Existing MAT programs may provide an opportunity to integrate effective, concurrent HIV prevention (e.g. PrEP) and counseling initiatives (Metzger et al., 2015; Metzger, Woody, \& O'Brien, 2010; Ratliff et al., 2013). Participants in MAT programs are generally engaged in care and are regularly taking medications, raising the possibility that these populations may be more willing to embrace biomedical interventions that involve continuous care. For populations who are CJ-involved in particular, regular medication provision may facilitate adherence. However, little research has examined the feasibility of combining the two in CJ settings (Brinkley-Rubinstein, Cloud, Drucker, \& Zaller, 2018; Rich, Bia, Altice, \& Feinberg, 2018).

The present study is the first to our knowledge to examine interest in PrEP among people who are incarcerated with OUD. This study is additionally novel in that it expands upon existing literature on PrEP willingness among men who are incarcerated to include women. We describe HIV risk perceptions, awareness of PrEP, and interest in PrEP among people who were incarcerated $(n=39)$ and are enrolled in a MAT program in Rhode Island. Given the need for both MAT and HIV prevention programs among people who use drugs, this research provides important information that can inform best practices to implement PrEP interventions in this population.

\section{Methods}

\subsection{Participants}

We conducted 39 semi-structured, qualitative interviews with people with OUD who were incarcerated within the Rhode Island Department of Corrections (RIDOC), the state's unified jail and prison system. Participants were screened for OUD at intake using Texas Christian University Drug Screen 5, which screens for mild to severe substance use disorder (Knight, Blue, Flynn, \& Knight, 2018). All participants were enrolled in a MAT program consisting of a methadone, suboxone, or naltrexone regimen. Inclusion criteria included current enrollment in the MAT program, being over 18 years old, and being able to read and write in English.

\subsection{Procedures}

Participants were recruited at the RIDOC during program group sessions by two research assistants who were trained in qualitative interviewing. The study was described and participants were able to confidentially sign up for the study and be contacted for an hour-long interview at a later time. All participants who signed up and were contacted completed the study. The sample was stratified to proportionally represent type of medication, whether uptake took place in the community or in the RIDOC, and facility at the RIDOC. We conducted interviews in intake, minimum security, medium security, and women's security facilities which are located in separate buildings on a unified campus. All interviews were conducted in a private room at the facility without correctional officers present. Interviews were digitally recorded and later professionally transcribed verbatim. All participants received \$25 that was deposited into their commissary account. The study was approved by the Miriam Hospital's Institutional Review Board and the RIDOC Medical Research Advisory Group.

\subsection{Measures}

Interviews in the Evaluating Medication for Addiction Treatment in a Unified Jail and Prison Setting (E-MAT) study assessed attitudes toward MAT, experiences in the MAT program, post-release substance use plans, program ethics, fentanyl perceptions, and HIV risk perceptions (Brinkley-Rubinstein et al., in press). The current paper focuses on questions related to HIV risk perceptions, prevention, and PrEP. The average interview length was $57 \mathrm{~min}$, and the range was 27 to $95 \mathrm{~min}$. Study protocols were developed ad hoc.

We queried the following questions: 1) Have you ever been tested for HIV before?; 2) Do you think you are at risk for HIV? (Prompt: why/ why not?); 3) Have you ever heard of pre-exposure prophylaxis (or PrEP) before? (Prompt: PrEP is a once daily medication that you can take to prevent HIV. What have you heard? Do you know anyone who has taken it?); 4) Would you be interested in taking PrEP, why/why not?, and 5) Can you tell me a little about your substance use? (Prompt: What kind of substances have you used? How often did you use? How long had you been using? What do you prefer to use most?). At the 
conclusion of the interview, participants were asked questions on demographic characteristics. The full protocol is available in Appendix A.

\subsection{Data analysis}

Analysis employed a general inductive approach, which allows for research to be divided into codes and themes in line with the research objectives and the questions asked during interviews (Thomas, 2006). Two coders (MP, KP) read through transcriptions looking for recurrent themes and patterns. Each theme was given a code, and codes were compiled in a codebook. Four coders (LBR, MP, KP, AT) initially performed preliminary coding and consolidated the codebook with any discrepancies being discussed and resolved among the coding team before final coding took place. Two coders (MP, AT) completed final coding in NVivo 12 with quality checks conducted on $20 \%$ of transcripts for thematic agreement. Thematic saturation was achieved was the final sample.

\section{Results}

In June through August 2018, we screened and enrolled 39 participants who answered questions related to HIV and PrEP. We have included an overview of participant demographics in Table 1. Participants were asked in an open-ended question to describe their substance use prior to their incarceration. Overall, 95\% $(n=37)$ reported using heroin, $74 \%(n=29)$ reported using prescription opioids, $53 \%$ $(n=21)$ reported using marijuana, $30 \%(n=12)$ reported benzodiazepine use, and $20 \%(n=8)$ reported using alcohol.

\subsection{PrEP awareness}

Most participants reported that they had not previously heard of PrEP. When asked if they had heard any information about PrEP, 77\% of participants $(n=30)$ revealed that they had never heard of PrEP before. The 23\% $(n=9)$ participants who had heard of PrEP provided varying information about where they had first learned of it. Three participants stated that they had heard about PrEP from television, one

Table 1

Characteristics of participants enrolled in the Evaluating Medication for Addictions Treatment (E-MAT) study.

\begin{tabular}{lll}
\hline Characteristics & $\mathrm{n}$ & $\%$ \\
\hline MAT type & & \\
$\quad$ Methadone & 20 & $51 \%$ \\
Suboxone & 18 & $46 \%$ \\
$\quad$ Naltrexone & 1 & $3 \%$ \\
Continued a prescription from outside the RIDOC v. Initiated while & & \\
$\quad$ incarcerated & & \\
Continued & 20 & $51 \%$ \\
Initiated & 19 & $49 \%$ \\
Gender & & \\
Male & 27 & $69 \%$ \\
Female & 12 & $31 \%$ \\
Race & & \\
Black & 2 & $5 \%$ \\
White & 32 & $82 \%$ \\
Mixed/bi-racial & 1 & $3 \%$ \\
Other & 4 & $10 \%$ \\
Ethnicity & & \\
Hispanic & 4 & $10 \%$ \\
Non-Hispanic & 35 & $90 \%$ \\
Sexual orientation & & \\
Straight & 34 & $87 \%$ \\
Gay & 2 & $5 \%$ \\
Bisexual & 3 & $8 \%$ \\
Highest education completed & & \\
Did not complete high school & 10 & $26 \%$ \\
Completed high school & 29 & $74 \%$ \\
\hline & &
\end{tabular}

reported from the community, one mentioned that they had first heard of it at the RIDOC when they met with a nurse for their medical intake while being booked into the facilitated, and four did not specify.

One female participant who had never heard of PrEP replied after receiving a summary: "I don't really think that - I don't need to, but that's interesting, because I've never heard of that." Another participant indicated that she had not heard of PrEP, but wished she had learned about it before: "I wish I knew that. I needed that."

Another participant who had heard previously of PrEP replied that he was planning to initiate PrEP after release with the assistance of a discharge planner:

I have. I'm not on PrEP medication. One of the doctors here is giving me a phone number to call when I get out. I guess I just found out that my Medicaid or Medicare will pay for PrEP. I didn't know that before. So, that's a good thing.

An additional participant mentioned that he had heard of PrEP as associated with risk perceptions:

Oh actually yes, I did hear something about that. Just the fact, like if you're shooting up and sharing needles, or if you have lots of unprotected sex I guess that's something that you would want to do.

While knowledge of PrEP varied across the sample, most participants had not heard of PrEP. Some participants who had not heard of PrEP stated that it would have been useful for them in the past, or that they were interested in learning more about it.

\subsection{HIV risk perceptions}

HIV risk perceptions varied across the sample with some participants reporting that they felt they were at risk, while others did not. Overall, $64 \%(n=25)$ of participants felt that they were not at risk, $23 \%$ of participants $(n=9)$ felt they were at risk, and the remaining $13 \%(n=5)$ were unsure if they were at risk for HIV acquisition. Many participants reported that at previous times they felt they were at risk based on behaviors (i.e. through sharing needles, sex without condoms), but that after MAT uptake, they no longer felt that they were at risk. Most participants connected their perceptions of personal HIV risk with their current sexual risk factors.

Of those who reported feeling at risk for HIV, most participants related their risk status to sexual behavior. One female participant, for instance, replied:

Actually, yes, it's always 50/50, because I tend to not use protection when I have sex. So yes, I'm at risk that way; but I haven't been using IV [intravenous] drugs. My veins are used up, so I can't even use IV if I wanted to. But having sex without protection - yes, I'm at risk.

Many participants reported that their risk status changed over time. While these participants reported that they did not feel currently at risk, they noted that their risk may have been higher in the past and may be subject to change. One participant stated that he had previously felt at risk for HIV, but no longer: "Well, I've gotten tested since my last time I used a needle, and I don't plan on using one again. And I don't have any blood to blood contact. I'm not sexually active right now." Another female participant responded: "No. Not any longer, I'm not. I'm not an IV user and I don't have multiple partners or anything, so I don't any longer think that I'm at risk for it."

Other participants noted that safer substance use or safer sex behaviors had previously protected them from HIV, and that they intended to continue these behaviors. For instance, one male participant answered:

Because I don't put myself in those situations no matter what. I don't care if I was dope sick for three days. I would go down to the CVS and buy a new needle before I would ever think about using somebody else's so I'm good. 
This participant definitively perceived that he was not at risk for HIV acquisition, and he could perceive no situations in which he would be in the future.

Most participants stated that they felt that at the time of the study, they did not perceive themselves to be at high risk for HIV acquisition upon release, and participants associated risk with behaviors related to sexual partnerships, condom use, and needle sharing. However, participants largely noted that over time, they had experienced changes in their perceived risk status (i.e. a recent decrease in risk), and some noted that their risk could change in the future. For example, one participant stated that he had previously felt at risk, but no longer:

If I was still going to - if I thought I was going to go out there and have a strong chance of relapsing, or I was going to be out there still messing around with drug addict women or - then yes, I would [take PrEP], but I'm not going to - I'm going to stay away from [women who use drugs.] I'm going to stay away from the drugs.

The participant stated that he perceived his HIV risk behavior as low despite being higher in the past when he was still using opioids.

Other participants did not report feeling that they were at risk for HIV acquisition based on behavior while incarcerated, though some noted that they may be at heightened risk after release. For instance, one 30-year-old female participant stated that her risk may be higher in the community after release: "because you never know what happens, you know." She described that she felt that she would likely abstain from using opioids and use adopt safer sex practices, but still felt some uncertainty surrounding her release and her anticipated behaviors.

\subsection{PrEP interest}

Interest in starting PrEP while incarcerated to prevent HIV acquisition after release varied, and PrEP interest was tied to HIV risk perceptions. Overall, $59 \%(n=23)$ of participants were not interested in taking PrEP, $31 \%(n=12)$ stated that they were interested in taking PrEP, and $10 \%$ were unsure. Of the 12 participants who were interested in PrEP uptake, 6 were female and 6 were male. All interested participants had graduated from high school. The age range of interested participants was 30-50 years old, with an average age of 35. Age of interested participants did not differ from the overall sample, which had an age range of 22-66 and a mean age of 37 . However, $50 \%(n=6)$ of the total female participants $(n=12)$ were interested in PrEP. Participants who felt that they were at risk for HIV discussed how PrEP may be useful. Some participants indicated that they would reassess their PrEP interest in the future should their behavior change.

Participants who no longer participated in risk behaviors noted that they would not be interested in PrEP. One female participant stated: "I don't need [PrEP]. I wouldn't need it. I don't shoot up anymore. I wouldn't shoot up anymore. I don't hang around with anybody. I just hang with my grandkids and my kids." The participant elaborated that while she used to inject drugs, she did not intend to do so after release and therefore did not perceive herself at risk for HIV acquisition. She also noted that her social network had changed, which also would impact her HIV risk status.

Some participants reported that while they did not currently feel at risk, they would be willing to take precautions through using PrEP should that status change. One male participant stated:

"Not myself, because I try not to put myself in high risk situations; but if I thought there was more of a chance - if I was going to be sleeping around, something like that, I would definitely [take PrEP] to have that extra layer of protection."

Others, such as one male participant, reported selectivity in sexual partners as a protective factor and a reason for his lack of interest in PrEP:

“Just because I don't use IVs, and if I'm ever sexually active, it's not with someone that's using IVs or was with someone that was in that type of situation. I mean, I guess anything could happen, but I don't put myself at a high risk level."

Among participants who were interested in PrEP, most noted either 1) current perceived risk or 2) feeling that they were not currently at risk, but preferring to take PrEP in case they ever found themselves in a situation where they would be at heightened risk for HIV acquisition. For example, one male participant noted that PrEP would be a useful tool in case of future relapse:

Good to have, yeah. Definitely. Like, if I was still using heroin and stuff like that, hell yeah, I'd want to take one every day to keep HIVfree. I think that's awesome.

Participants who indicated interest in PrEP often expressed sentiments such as feeling that it was impossible to predetermine risk behaviors, and using PrEP could protect them ahead of time. One male participant noted:

Yeah, sure. Absolutely. Anything would be good, because you never know. Someone can accidentally use your stuff, and they could be sick. Not that I'm around people that do that, but that's something I'd be interested in. I don't see why anybody wouldn't. That's a way of protecting yourself ahead of time. Yeah, I'd be interested in that.

Other participants discussed factors such as having sex with multiple partners or condomless sex as reasons for interest in PrEP. One male participant commented:

Because I wouldn't feel like I would want to get in a relationship, and have that responsibility, because I'm homeless. But I do like compassion and affection and having a trustworthy being, a friend, but there's still chances of catching HIV if you're with the wrong person.

In summary, interest in PrEP was low but varied across participants, with those reporting potential for heightened risk as being more likely to express interest in taking PrEP.

\subsection{Barriers to PrEP use}

\subsubsection{Fears of side effects}

Some participants who either did not want to take PrEP or were unsure about taking it discussed barriers to PrEP use. One participant outlined how while she was unsure if she was interested, the side effects could dissuade her from using PrEP. She stated: "I don't know. Maybe, but I - not really, no, because I heard all the side effects it has. I mean, just to prevent something, I'm going to add like six more side effects to me? No."

Discussions of side effects often referred to distrust of the medical institutions general, including of both medical services and pharmaceuticals. For example, another male participant commented:

I'm all set with testing a new drug that you don't know the side effects, you don't know what's going on. A lot of stuff like that ends up causing cancer and shit that you don't know a lot about. I just use a condom and try to do my best to stay away from it.

The participant was not interested in PrEP based on the belief that medications intending to help could have adverse side effects, and that pharmaceutical companies distributing the medication may not fully understand the side effects of the medication. The participant preferred methods which he felt familiar with and did not anticipate health risks for taking them. Side effects were therefore an important concern among those included in the sample.

\subsubsection{Reluctance to add medications to regimen}

An additional barrier reported by participants was a general reluctance to add more medications to their existing medication regimen. One participant claimed that rather than adding medications, he would prefer to change the underlying behavior that led to his risk status. He claimed: "I don't really take meds. I just don't. And I don't want to take - 
for me, it would make more sense to stop sharing needles than to take a pill." He therefore felt that he was uninterested in PrEP because he did not want to take more medications. Some participants expressed similar sentiments that while PrEP may be useful, they preferred not to take because they did not like to take medications. One male participant stated:

I mean, yeah. I mean yes and no because I don't like to take medications but yeah I mean if it ever came to a point where I was going to start engaging in situations where I know I might put myself at risk for HIV then yea. Until then, I see no use for it.

Another participant noted that he would potentially be interested in PrEP, but he was initially skeptical of adding further medications in addition to his MAT treatment. He noted that while he would consider PrEP, he would need to learn more about it before taking it daily:

"I'd have to look into it a little bit before I start taking all these crazy medicines." The participant was uncertain about adding medications to his regimen without prior knowledge, alluding to the fact that he perceived taking excessive medications as potentially harmful.

\subsubsection{Worries about adherence and de-prioritization of PrEP}

Another potential barrier that was discussed alongside interest in taking PrEP was participants' worries about their ability to adhere to a PrEP regimen. Some participants noted that they would be unlikely to take a medication every day, or that if they were to return to a situation where they were at risk, they would likely deprioritize medication. One participant cited that she would likely only benefit from PrEP if she were injecting drugs again, but that if she were using drugs, she would likely not think to take her medication. She stated: "I mean I don't know. Like I wouldn't be taking it if I wasn't using so. If I was using I probably wouldn't be taking it anyway because I don't take my medication like I'm supposed to so who knows." Adherence was an important barrier for many to taking PrEP.

\section{Discussion}

To our knowledge, this is the first study to examine perceptions of PrEP among people who were incarcerated and receiving MAT for opioid use disorders. Knowledge of and interest in PrEP varied widely among people who were incarcerated and enrolled in a MAT program. Most participants demonstrated low knowledge of PrEP. After PrEP was briefly explained to participants ("PrEP is a once daily medication that you can take to prevent HIV"), $31 \%(n=12)$ of participants stated that they would be interested in taking PrEP to prevent HIV acquisition after release. While many participants were not interested in taking PrEP at the time of interview, some explained that they would like more information about PrEP to inform their decision.

Participants in this study conceptualized their HIV risk status as fluid across time. Some participants stated that while they would not consider PrEP to be viable for themselves at the time of the interview, they would consider initiating PrEP should their risk status change. This finding corroborates previous literature showing that individuals who are at risk for HIV may demonstrate higher PrEP acceptability during "seasonal" shifts to higher risk behavior (Hojilla et al., 2016; Rolle et al., 2017; Underhill et al., 2018). The most common barriers to PrEP uptake included fears of side effects, adherence, and reluctance to take and distrust of medications generally. In addition, many participants may not conceptualize their current HIV risk as high because 1) they are not engaging in risk behaviors while incarcerated and 2) because they are engaged in a treatment program with the goal of de-escalating injection drug use post-release. This latter point may also explain why many participants also solely considered their HIV risk relative to their sexual (and not drug use) behaviors.

Given that participants are enrolled in programs designed to enhance medication adherence and that some participants were interested in PrEP, these settings may provide opportunities for intervention. MAT programs in CJ settings may serve as facilitators to improved outcomes in the "PrEP continuum of care" through increased PrEP awareness, uptake, as well as adherence and retention (Nunn et al., 2017). People who are incarcerated-and those in OUD treatment programs-should be provided with comprehensive information about PrEP, which may increase medication uptake and reduce HIV acquisition upon release. Further research is needed in this area. Many participants further noted that they were interested in minimizing HIV risk behaviors instead of uptaking PrEP. MAT settings may also incorporate information on HIV risk reduction overall to support participants in making informed decisions about HIV risk management options.

For those interested in PrEP, comprehensive discharge planning and education should also be employed to improve adherence. Additionally, all people who are incarcerated-regardless of their current risk status-should be provided with information about where to access PrEP in the community should their intention to initiate PrEP change later. This would closely correspond with an approach recommended by the World Health Organization (WHO). The WHO has suggested that anyone belonging to a population that has an increased burden of HIV (defined as having a HIV incidence equal to or higher than 3 per 100 person-years) is at substantive risk and eligible for PrEP, which would broadly include people who are CJ-involved (World Health Organization, 2015). Further, future research is warranted in the areas of implementation research surrounding increasing knowledge of and interest in PrEP. Future research should also explore building a continuum of PrEP from prison/jail to communities.

The present study has several limitations. First, the sample was mostly White, which does not represent people who are incarcerated broadly. Second, HIV risk perception relied on self-report in recorded interviews and may be subject to recall bias, inaccurate perceptions of risk, or social desirability bias. Third, the sample only included participants with OUD who were enrolled in a MAT program, and did not include those who had refused MAT during incarceration, or those who were not eligible for the program. It is possible that answers may have differed from those who were not enrolled in MAT based on willingness to take MAT. With these limitations present, the study intended to provide a snapshot of those incarcerated and enrolled in a correctional MAT program in Rhode Island and may not be generalizable to larger populations.

\section{Conclusions}

Findings explored how participants had low knowledge of PrEP but that some (31\%) were interested in PrEP after learning more about it. While PrEP interest varied, some participants expressed that they would be interested in learning further about PrEP or exploring uptake. Discharge planning and correctional health education should provide comprehensive information on and linkage to PrEP if interested for people who are incarcerated. PrEP interest related to perceived HIV risk factors. Potential barriers included side effects, adherence, and reluctance to take medications in general. Given the high risk for HIV acquisition upon release and demonstrated interest, MAT programs for people who are criminal justice (CJ) involved may serve as useful linkage spaces to PrEP information, access, and retention.

Supplementary data to this article can be found online at https:// doi.org/10.1016/j.jsat.2019.08.015.

\section{Funding}

The study was funded by the National Institute on Drug Abuse (NIDA) under grant number 5R21DA044443-02.

\section{References}

Anderson, P. L., Glidden, D. V., Liu, A., Buchbinder, S., Lama, J. R., Guanira, J. V., \& Veloso, V. G. (2012). Emtricitabine-tenofovir concentrations and pre-exposure 
prophylaxis efficacy in men who have sex with men. Sci. Transl. Med. 4(151), 151 ra125.

Baeten, J. M., Donnell, D., Ndase, P., Mugo, N. R., Campbell, J. D., Wangisi, J., \& Ronald, A. (2012). Antiretroviral prophylaxis for HIV prevention in heterosexual men and women. N. Engl. J. Med. 367(5), 399-410.

Brinkley-Rubinstein, L., Cloud, D., Drucker, E., \& Zaller, N. (2018). Opioid use among those who have criminal justice experience: Harm reduction strategies to lessen HIV risk. Curr. HIV/AIDS Rep. 15(3), 255-258.

Brinkley-Rubinstein, L., Peterson, M., Arnold, T., Nunn, A. S., Beckwith, C. G., Castonguay, B., \& Chan, P. A. (2018). Knowledge, interest, and anticipated barriers of pre-exposure prophylaxis uptake and adherence among gay, bisexual, and men who have sex with men who are incarcerated. PLoS One, 13(12), e0205593.

Brinkley-Rubinstein, L., Peterson, M., Clarke, J., Macmadu, A., Truong, A., Pognon, K., ... Rich, J. (2019). The benefits and implementation challenges of the first state-wide comprehensive medication for addictions program in a unified jail and prison setting. Drug Alcohol Depend. (in press).

Centers for Disease Control and Prevention, \& US Public Health Service (2018). Preexposure prophylaxis for the prevention of HIV infection in the United States-2017 update: A clinical practice guideline. Atlanta: CDC.

Choopanya, K., Martin, M., Suntharasamai, P., Sangkum, U., Mock, P. A., Leethochawalit, M., \& Chuachoowong, R. (2013). Antiretroviral prophylaxis for HIV infection in injecting drug users in Bangkok, Thailand (the Bangkok Tenofovir Study): A randomised, double-blind, placebo-controlled phase 3 trial. Lancet, 381(9883), 2083-2090.

Dauria, E. F., Tolou-Shams, M., Levine-Murray, A., Monique Lipman, A. S. W., Comfort, M., \& Christopoulos, K. (2017). "We need this!" PrEP awareness and acceptability among women involved in the criminal justice system. Oral presentation. 12th international conference on HIV treatment and prevention adherence, Miami, FL.

Escudero, D. J., Kerr, T., Wood, E., Nguyen, P., Lurie, M. N., Sued, O., \& Marshall, B. D. (2015). Acceptability of HIV pre-exposure prophylaxis (PrEP) among people who inject drugs (PWID) in a Canadian setting. AIDS Behav. 19(5), 752-757.

Gordon, M. S., Kinlock, T. W., Schwartz, R. P., Fitzgerald, T. T., O'Grady, K. E., \& Vocci, F. J. (2014). A randomized controlled trial of prison-initiated buprenorphine: Prison outcomes and community treatment entry. Drug Alcohol Depend. 142, 33-40.

Grant, R. M., Anderson, P. L., McMahan, V., Liu, A., Amico, K. R., Mehrotra, M., \& Buchbinder, S. (2014). Uptake of pre-exposure prophylaxis, sexual practices, and HIV incidence in men and transgender women who have sex with men: A cohort study. Lancet Infect. Dis. 14(9), 820-829.

Guise, A., Albers, E. R., \& Strathdee, S. A. (2017). 'PrEP is not ready for our community, and our community is not ready for PrEP': Pre-exposure prophylaxis for HIV for people who inject drugs and limits to the HIV prevention response. Addiction, 112(4), 572-578.

Hojilla, J. C., Koester, K. A., Cohen, S. E., Buchbinder, S., Ladzekpo, D., Matheson, T., \& Liu, A. Y. (2016). Sexual behavior, risk compensation, and HIV prevention strategies among participants in the San Francisco PrEP demonstration project: A qualitative analysis of counseling notes. AIDS Behav. 20(7), 1461-1469.

Khan, M. R., Behrend, L., Adimora, A. A., Weir, S. S., White, B. L., \& Wohl, D. A. (2011). Dissolution of primary intimate relationships during incarceration and implications for post-release HIV transmission. J. Urban Health, 88(2), 365-375.

Kirby, T., \& Thornber-Dunwell, M. (2014). Uptake of PrEP for HIV slow among MSM. Lancet, 383(9915), 399-400.

Knight, D. K., Blue, T. R., Flynn, P. M., \& Knight, K. (2018). The TCU drug screen 5: Identifying justice-involved individuals with substance use disorders. J. Offender Rehabil. 57(8), 525-537.

Kuo, I., Olsen, H., Patrick, R., Phillips, G., II, Magnus, M., Opoku, J., \& Greenberg, A. (2016). Willingness to use HIV pre-exposure prophylaxis among community-recruited, older people who inject drugs in Washington, DC. Drug Alcohol Depend. 164, $8-13$.

Maruschak, L. M. (2012). HIV in prisons, 2001-2010. AIDS, 20(25.10), 1-11.

Mathers, B. M., Degenhardt, L., Phillips, B., Wiessing, L., Hickman, M., Strathdee, S. A., \& Mattick, R. P. (2008). Global epidemiology of injecting drug use and HIV among people who inject drugs: A systematic review. Lancet, 372(9651), 1733-1745.

Mayer, K. H., Chan, P. A., Patel, R., Flash, C. A., \& Krakower, D. S. (2018). Evolving models and ongoing challenges for HIV pre-exposure prophylaxis implementation in the United States. J. Acquir. Immune Defic. Syndr. 77(2), 119 (1999).

Metzger, D. S., Donnell, D., Celentano, D. D., Jackson, J. B., Shao, Y., Aramrattana, A., .. \& Chawarski, M. (2015). Expanding substance use treatment options for HIV prevention with buprenorphine-naloxone: HIV prevention trials network 058 (HPTN 058). J. Acquir. Immune Defic. Syndr., 68(5), 554.

Metzger, D. S., Woody, G. E., \& O'Brien, C. P. (2010). Drug treatment as HIV prevention: A research update. J. Acquir. Immune Defic. Syndr. 55(Suppl. 1), S32.

Morrow, K. M., \& The Project START Study Group 1 (2009). HIV, STD, and hepatitis risk behaviors of young men before and after incarceration. AIDS Care, 21(2), 235-243.

Nunn, A. S., Brinkley-Rubinstein, L., Oldenburg, C. E., Mayer, K. H., Mimiaga, M., Patel, R., \& Chan, P. A. (2017). Defining the HIV pre-exposure prophylaxis care continuum. AIDS (London, England), 31(5), 731.

Peterson, M., Nowotny, K., Dauria, E., Arnold, T., \& Brinkley-Rubinstein, L. (2019). Institutional distrust among gay, bisexual, and other men who have sex with men as a barrier to accessing pre-exposure prophylaxis (PrEP). AIDS Care, 31(3), 364-369.

Ratliff, E. A., McCurdy, S. A., Mbwambo, J. K., Lambdin, B. H., Voets, A., Pont, S., \& Kilonzo, G. P. (2013). An overview of HIV prevention interventions for people who inject drugs in Tanzania. Adv. Prev. Med. 2013.

Rich, J. D., McKenzie, M., Larney, S., Wong, J. B., Tran, L., Clarke, J., \& Zaller, N. (2015) Methadone continuation versus forced withdrawal on incarceration in a combined US prison and jail: A randomised, open-label trial. Lancet, 386(9991), 350-359.

Rich, K. M., Bia, J., Altice, F. L., \& Feinberg, J. (2018). Integrated models of care for individuals with opioid use disorder: How do we prevent HIV and HCV? Curr. HIV/ AIDS Rep. 15(3), 266-275.

Rolle, C. P., Rosenberg, E. S., Siegler, A. J., Sanchez, T. H., Luisi, N., Weiss, K., ... Kelley, C. F. (2017). Challenges in translating PrEP interest into uptake in an observational study of young black MSM. J. Acquir. Immune Defic. Syndr. 76(3), 250-258.

Sherman, S. G., Schneider, K. E., Park, J. N., Allen, S. T., Hunt, D., Chaulk, C. P., \& Weir, B. W. (2019). PrEP awareness, eligibility, and interest among people who inject drugs in Baltimore, Maryland. Drug Alcohol Depend. 195, 148-155.

Shrestha, R., Altice, F. L., Huedo-Medina, T. B., Karki, P., \& Copenhaver, M. (2017) Willingness to use pre-exposure prophylaxis (PrEP): An empirical test of the information-motivation-behavioral skills (IMB) model among high-risk drug users in treatment. AIDS Behav. 21(5), 1299-1308.

Shrestha, R., \& Copenhaver, M. (2018). Exploring the use of pre-exposure prophylaxis (PrEP) for HIV prevention among high-risk people who use drugs in treatment. Front. Public Health, 6.

Stein, M., Thurmond, P., \& Bailey, G. (2014). Willingness to use HIV pre-exposure prophylaxis among opiate users. AIDS Behav. 18(9), 1694-1700.

Stekler, J. D., Ure, G., O'Neal, J. D., Lane, A., Swanson, F., Maenza, J., \& Golden, M. R. (2016). Performance of determine combo and other point-of-care HIV tests among Seattle MSM. J. Clin. Virol. 76, 8-13.

Thomas, D. R. (2006). A general inductive approach for analyzing qualitative evaluation data. Am. J. Eval. 27(2), 237-246.

Underhill, K., Guthrie, K. M., Colleran, C., Calabrese, S. K., Operario, D., \& Mayer, K. H. (2018). Temporal fluctuations in behavior, perceived HIV risk, and willingness to use pre-exposure prophylaxis (PrEP). Arch. Sex. Behav. 47(7), 2109-2121.

Walters, S. M., Reilly, K. H., Neaigus, A., \& Braunstein, S. (2017). Awareness of preexposure prophylaxis (PrEP) among women who inject drugs in NYC: The importance of networks and syringe exchange programs for HIV prevention. Harm Reduct. J. 14(1), 40.

Wohl, D. A. (2016). HIV and mass incarceration: Where infectious diseases and social justice meet. N. C. Med. J. 77(5), 359-364.

World Health Organization (2015). Guideline on when to start antiretroviral therapy and on pre-exposure prophylaxis for HIV, web supplement: Annex 2: Evidence to decision-making tables and supporting evidence (no. WHO/HIV/2015.36). World Health Organization.

Ya-lin, A. H., Zhu, W., Smith, D. K., Harris, N., \& Hoover, K. W. (2018). HIV preexposure prophylaxis, by race and ethnicity-United States, 2014-2016. Morb. Mortal. Wkly Rep. 67(41), 1147.

Zaller, N., McKenzie, M., Friedmann, P. D., Green, T. C., McGowan, S., \& Rich, J. D. (2013). Initiation of buprenorphine during incarceration and retention in treatment upon release. J. Subst. Abus. Treat. 45(2), 222-226. 